\title{
Cyclic Inequality Forms with Power 1/2,1/3
}

\author{
Pham Van Quoc* \\ High School for Gifted Student, VNU University of Science
}

Received 14 June 2017

Accepted 19 September 2017

Abstract: The purpose of this paper is to establish inequalities between two terms

$$
\begin{gathered}
F=\sum_{i=1}^{n} \sqrt{a x_{i}^{2}+b x_{i} x_{i+1}+c x_{i+1}^{2}+d x_{i}+e x_{i+1}+d} ; \\
G=\sum_{i=1}^{n} \sqrt[3]{a x_{i}^{3}+b x_{i}^{2} x_{i+1}+c x_{i} x_{i+1}^{2}+d x_{i+1}^{3}},
\end{gathered}
$$

and $\sum_{i=1}^{n} x_{i}$ for a sequence of cyclic positive real numbers $\left(x_{i}\right)_{i=1}^{n+1}$ with $x_{n+1}=x_{1}$.

Keywords: Cyclic inequality, power 1/2,1/3.

\section{Introduction}

Let $x_{1}, x_{2}, \ldots, x_{n}$ be sequence of positive number satisfying $x_{l}=x_{n}$. The cyclic inequalities of $\mathrm{n}$ variables have a form

$$
S\left(x_{1}, x_{2}, \ldots, x_{n}\right) \geq 0,
$$

where $S$ is a function of $n$ variables, valued in $\mathbf{R}$. In [2] author considers the function

$$
f(x, y, z)=\frac{x}{y+z}
$$

to establish a cyclic inequality

$$
S\left(x_{1}, x_{2}, \ldots, x_{n}\right)=\frac{x_{1}}{x_{2}+x_{3}}+\frac{x_{2}}{x_{3}+x_{4}}+\ldots+\frac{x_{n-1}}{x_{n}+x_{1}}+\frac{x_{n}}{x_{1}+x_{2}}-\frac{n}{2},
$$

and conjectures that $S\left(x_{1}, x_{2}, \ldots, x_{n}\right) \geq 0$. On the other hand, the well-known inequality

$$
\sqrt{a^{2}+x^{2}}+\sqrt{b^{2}+y^{2}}+\sqrt{c^{2}+z^{2}} \geq \sqrt{(a+b+c)^{2}+(x+y+z)^{2}}
$$

\footnotetext{
Tel.: 84- 888558881.

Email: quocpv@gmail.com

https//doi.org/ 10.25073/2588-1124/vnumap.4209
} 
is showed in [7, chapter V] with its corollary $\sqrt{a^{2}+b^{2}}+\sqrt{b^{2}+c^{2}}+\sqrt{c^{2}+a^{2}} \geq \sqrt{2}(a+b+c)$. Some inequalities are "similar" but they are in opposite side

$$
\begin{gathered}
\sqrt{a^{2}+5 a b+4 b^{2}}+\sqrt{b^{2}+5 b c+4 c^{2}}+\sqrt{c^{2}+5 c a+4 a^{2}} \leq 3(a+b+c) \\
\sqrt{a^{2}+b c}+\sqrt{b^{2}+c a}+\sqrt{c^{2}+a b} \leq \frac{3}{2}(a+b+c)
\end{gathered}
$$

in [5, chapter 5].

In this paper, instead of using the function $f$ in (1.1), we use the the functions (1.2), (1.3) and (1.4) to establish cyclic inequalities by comparing the functions

$$
\begin{gathered}
F\left(x_{1}, x_{2}, \ldots, x_{n}\right)=\sum_{i=1}^{n} \sqrt{a x_{i}^{2}+b x_{i} x_{i+1}+c x_{i+1}^{2}} \text { or } \\
G\left(x_{1}, x_{2}, \ldots, x_{n}\right)=\sum_{i=1}^{n} \sqrt[3]{a x_{i}^{3}+b x_{i}^{2} x_{i+1}+c x_{i} x_{i+1}^{2}+d x_{i+1}^{3}}
\end{gathered}
$$

with a function of $\sum_{i=1}^{n} x_{i}$, where $x_{n+1}=x_{1}$.

The main way to approach the problem is firstly to establish an inequality between $\sqrt{a x^{2}+b x y+c y^{2}}$ and $\alpha \mathrm{x}+\beta \mathrm{y}$ and then to give a condition on $\Delta=b^{2}-4 a c$ for which we obtain the result

$$
F=\sqrt{a+b+c} \geq \sum_{i=1}^{n} x_{i} \text { or } F=\sqrt{a+b+c} \leq \sum_{i=1}^{n} x_{i}
$$

depending on the sign of $\Delta$. We also consider a general problem with the expression $A(x ; y)=a x^{2}+b x y+c y^{2}+d x+e x+f$. By reducing it into a simpler form $A(x ; y)=a x^{2}+b x y+c y^{2}+d$, we get the same inequality in case $\Delta>0$. For general $n$, it is still an open problem.

For the expression $G$, by using the similar idea of proving $F$, we try to drive an expression

$$
a x^{3}+b x^{2} y+c x y^{2}+d y^{3}=(\alpha x+\beta y)^{3}+(\gamma x+\delta y)(x-y)^{2},
$$

and depending on sign of $\gamma, \delta$ we have the comparison between $G$ and $\sum x_{i}$. This paper is organised as follows. In section 2 we consider cyclic inequality with power $\frac{1}{2}$ by using the function $f(x, y)=a x^{2}+b x y+c y^{2}+d x+e y+f$. Section 3 deals with cyclic inequality with power $\frac{1}{3}$ with the function $f(x, y)=a x^{3}+b x^{2} y+c x y^{2}+d y^{3}$. 


\section{Cyclic inequality with power $\frac{1}{2}$}

First at all, we establish an inequality for $\sqrt{a x^{2}+b x y+c y^{2}+d}$. To start with, we will find the condition such that the expression $\sqrt{a x^{2}+b x y+c y^{2}+d}$ is defined for all $x, y \geq 0$.

Put $A(x ; y)=a x^{2}+b x y+c y^{2}+d$ and $\Delta=b^{2}-4 a c$.

Lemma 2.1 $A(x ; y) \geq 0$ for all $\mathrm{x}, \mathrm{y} \geq 0$ if and only if one of two following conditions holds

i) $a, c, b, d \geq 0$;

ii) $a, c, d \geq 0, b<0$ and $\Delta \leq 0$.

Proof. Since $A(0,0)=d$, it follows that $d \geq 0$. Writing $A(x, y)=y^{2}\left(a t^{2}+b t+c\right)+d$ with $t=\frac{x}{y}$ yields $a t^{2}+b t+c \geq 0$ for all $t \geq 0$. Indeed, if there is $t_{0}$ such that $a t_{0}^{2}+b t_{0}+c<0$ then we let $y \rightarrow \infty$ to get a contradiction. The case $a=0$ is trivial, so let $a \neq 0$. From the property $a t^{2}+b t+c \geq 0$ for all $t \geq 0$ it follows $a, c \geq 0$. Hence, if $b \geq 0$ we have $i$ ). Otherwise, If $b<0$, we use the expression $A(x ; y)=y^{2} a\left(\left(t+\frac{b}{2 a}\right)^{2}-\frac{\Delta}{4 a^{2}}\right)$ then with $t=-\frac{b}{2 a}>0$ we see that $A(x ; y) \geq 0$ is equivalent to $\Delta \leq 0$. Thus we have $i$ ). The proof is complete.

Remark 2.2 In both conditions we have $a+b+c \geq 0$. If $a+b+c=0$ then the condition becomes $a=c=-\frac{b}{2}=0$.

For the sake of simplicity, from now on we assume $a>0$. Firstly, we consider the case $d=0$.

Lemma 2.3 Let $a, b, c$ be real numbers such that $a+b+c>0$. There exist $\alpha, \beta, \gamma$ such that $\alpha+\beta>0$ and

$$
a x^{2}+b x y+c y^{2}=(\alpha x+\beta y)^{2}+\gamma(x-y)^{2} .
$$

Proof. Developing the desired equality and comparing coefficients of both sides we have the equation

$$
\left\{\begin{array}{c}
\alpha^{2}+\beta=a \\
\beta^{2}+\gamma=c \\
2 \alpha \beta-2 \gamma=b
\end{array}\right.
$$

Summing these equations, we get $(\alpha+\beta)^{2}=a+b+c$. Thus, $\alpha+\beta=\sqrt{a+b+c}$. Now, subtracting the first and the second equation in (2.1) we have $\alpha^{2}-\beta^{2}=a-c$ which follows $\alpha-\beta=\frac{a-c}{\sqrt{a+b+c}}$. Hence

$$
\alpha=\frac{2 a+b}{2 \sqrt{a+b+c}}, \beta=\frac{2 c+b}{2 \sqrt{a+b+c}} .
$$

Substituting $\alpha, \beta$ into the last equation in (2.1) yields 


$$
\gamma=\frac{4 a c-b^{2}}{4(a+b+c)}
$$

We have the proof.

Theorem 2.4 Let $a, b, c$ be real numbers such that $a, c, a+b+c>0$. For any sequence of real positive numbers $x_{1}, x_{2}, \ldots, x_{n+1}$ with $x_{n+1}=x_{1}$ we have

i) if $\Delta \leq 0$ then $F\left(x_{1}, x_{2}, \ldots, x_{n}\right) \geq \sqrt{a+b+c} \sum_{i=1}^{n} x_{i}$;

ii) if $\Delta>0, a, b, c \geq 0$ then $F\left(x_{1}, x_{2}, \ldots, x_{n}\right) \leq \sqrt{a+b+c} \sum_{i=1}^{n} x_{i}$.

The equality occurs if and only if $x_{1}=x_{2}=\ldots=\xi_{V}$.

Proof. Applying Lemma

$$
\sqrt{a x_{i}^{2}+b x_{i} x_{i+1}+c x_{i+1}^{2}}=\sqrt{\left(\alpha x_{i}+\beta x_{i+1}\right)^{2}+\gamma\left(x_{i}-x_{i+1}\right)^{2}},
$$

for $i=1,2, \ldots, n, x_{n+1}=x_{1}$.

i) It is clear that $\Delta \leq 0$ is equivalent to $\gamma \geq 0$ or $\sqrt{a x^{2}+b x y+c y^{2}} \geq|\alpha x+\beta y|$. Therefore

$$
\begin{aligned}
F\left(x_{1}, x_{2}, \ldots, x_{n}\right) & \geq \sum_{i=1}^{n}\left|\alpha x_{i}+\beta x_{i+1}\right| \geq\left|\sum_{i=1}^{n}(\alpha+\beta) x_{i}\right| \\
& \geq \sqrt{a+b+c} \sum_{i=1}^{n} x_{i} .
\end{aligned}
$$

We have the first conclusion.

ii) The second is easy since $\alpha, \beta \geq 0, \gamma<0$.

Now, we study the problem in a more general context. Firstly, we consider the following proposition:

Proposition 2.5. The relation

$$
a x^{2}+b x y+c y^{2}+d x+e y+f \geq 0
$$

holds for all real values of $\mathrm{x}$ and $\mathrm{y}$ if and only if one of the following items $\mathrm{i}$ ), ii), iii) or iv) is satisfied
i) $a>0,\left|\begin{array}{cc}2 a & b \\ b & 2 c\end{array}\right|>0,\left|\begin{array}{ccc}2 a & b & d \\ b & 2 c & e \\ d & e & 2 f\end{array}\right|>0$
ii) $a>0,\left|\begin{array}{cc}2 a & b \\ b & 2 c\end{array}\right|=0,\left|\begin{array}{cc}2 a & b \\ d & e\end{array}\right|=0,\left|\begin{array}{cc}2 a & d \\ d & 2 f\end{array}\right|>0$,
iii) $a=b=d=0, c>0,\left|\begin{array}{cc}2 c & e \\ e & 2 f\end{array}\right|>0$,
iv) $a=b=c=d=e=0, f>0$.

Proof. See [6], chapter 11. 


\section{Denote}

$$
A(x ; y)=a x^{2}+b x y+c y^{2}+d x+e y+f, a \neq 0, \Delta=b^{2}-4 a c .
$$

By changing variables $x=X+m, y=Y+n$ then $A(x ; y)=a X^{2}+b X Y+c Y^{2}+D X+E Y+F$ where $D=2 a m+b n+d, E=2 c n+b m+e, F=a m^{2}+b m n+c n^{2}+d m+e n+f$. It depends on $\Delta \neq 0$ or $\Delta=0$ we can choose $m, n$ such that $D=E=0$ and $A(x ; y)$ transforms into one of the two types:

i) $A_{l}(x ; y)=a x^{2}+b x y+c y^{2}+d$;

ii) $A_{2}(x ; y)=a x^{2}+b y+c$.

It is not difficult to give some conditions on $a, b, c, d, e, f$ such that $A(x ; y)=0$ for all $x, y \geq 0$. However, these conditions are very complicated. So, for simple, from now on, we only consider the cases of $A_{I}(x ; y), A_{2}(x ; y) \geq 0$ for all $x, y \geq 0$.

For the case $i: A(x ; y)=a x^{2}+b x y+c y^{2}+d$. We now consider the case $d>0$. As in Lemma 1 , to make sure $A(x ; y) \geq 0$ for all $x, y \geq 0$ we need conditions: $a, b, c, d \geq 0$ or $a, c, d \geq 0, b<0, \Delta=b^{2}-4 a c \leq 0$. Without loosing of generality we can assume $d=1$. Then,

$$
F\left(x_{1}, x_{2}, \ldots, x_{n}\right)=\sum_{i=1}^{n} \sqrt{a x_{i}^{2}+b x_{i} x_{i+1}+c x_{i+1}^{2}+1},\left(x_{n+1}=x_{1}\right) .
$$

Theorem 2.6 Let a,b,c be real numbers such that $a, c, d, a+b+c>0, \Delta=b^{2}-4 a c \leq 0$. For any real positive number $x_{1}, x_{2}, \ldots, x_{n}$ we have

$$
F\left(x_{1}, x_{2}, \ldots, x_{n}\right) \geq \sqrt{(a+b+c)\left(\sum_{i=1}^{n} x_{i}\right)^{2}+n^{2}} .
$$

The equality occurs if and only if $x_{1}=x_{2}=\ldots=x_{n}$.

Proof. Since $\Delta \leq 0, \gamma \geq 0$. Applying Lemma 2 we have $\sqrt{a x_{i}^{2}+b x_{i} x_{i+1}+c x_{i+1}^{2}+1} \geq \sqrt{\left(\alpha x_{i}+\beta x_{i+1}\right)^{2}+1}$. Therefore,

$$
\begin{aligned}
F \quad \geq & \sum_{i=1}^{n} \sqrt{\left(\alpha x_{i}+\beta x_{i+1}\right)^{2}+1} \\
& \geq \sqrt{\left(\sum_{i=1}^{n} \alpha x_{i}+\beta x_{i+1}\right)^{2}+(1+\ldots+1)^{2}} \\
& =\sqrt{(\alpha+\beta)^{2}\left(\sum_{i=1}^{n} x_{i}\right)^{2}+n^{2}} \\
& =\sqrt{(a+b+c)\left(\sum_{i=1}^{n} x_{i}\right)^{2}+n^{2}} .
\end{aligned}
$$

And we have the conclution. 
Now, consider the case $\Delta>0$, to estimate $F$, we need some more conditions on $a, b, c$ and it becomes very complicate if $n \geq 4$. For $n=2,3$ we have results:

Proposition 2.7 i) If $a, c>0, b \geq a+c$ then

$$
F_{2}(x ; y) \leq \sqrt{(a+b+c)(x+y)^{2}+4} .
$$

ii) If $a, c>0, b \geq 2 a+2 c$ then

$$
F_{3}(x ; y ; z) \leq \sqrt{(a+b+c)(x+y+z)^{2}+9} .
$$

The equality holds iff $x=y$ in $i$ ), or $x=y=z$ in ii).

Proof. $i$ ) We have

$$
\begin{aligned}
F_{2}(x ; y) & =\sqrt{(\alpha x+\beta y)^{2}+\gamma(x-y)^{2}+1}+\sqrt{(\alpha y+\beta x)^{2}+\gamma(y-x)^{2}+1} \\
& \leq \sqrt{2\left((\alpha x+\beta y)^{2}+(\alpha y+\beta x)^{2}+2 \gamma(x-y)^{2}+2\right)} .
\end{aligned}
$$

Moreover,

$$
\begin{aligned}
& (\alpha+\beta)^{2}(x+y)^{2}-2(\alpha x+\beta y)^{2}-2(\beta x+\alpha y)^{2}-4 \gamma(x-y)^{2} \\
& =(x-y)^{2}\left(-\alpha^{2}+2 \alpha \beta-\beta^{2}-4 \gamma\right)=(x-y)^{2}(-a+b-c) \geq 0
\end{aligned}
$$

since $b \geq a+c$. Hence,

$$
F_{2}(x ; y) \leq \sqrt{(\alpha+\beta)^{2}(x+y)^{2}+4}=\sqrt{(a+b+c)(x+y)^{2}+4} .
$$

ii) Similarly,

$$
F_{2}(x ; y) \leq \sqrt{3\left((\alpha x+\beta y)^{2}+(\alpha y+\beta z)^{2}+(\alpha z+\beta x)^{2}+\gamma K+3\right)} .
$$

where $K=(x-y)^{2}+(y-z)^{2}+(z-x)^{2} \geq 0$. Noting that

$$
\begin{gathered}
(\alpha+\beta)^{2}(x+y+z)^{2}-3(\alpha x+\beta y)^{2}-3(\alpha y+\beta z)^{2}-3(\alpha z+\beta x)^{2}-3 \gamma K \\
\left(-\alpha^{2}+\alpha \beta-\beta^{2}-3 \gamma\right) K=\left(-a+\frac{b}{2}-c\right) K \geq 0 .
\end{gathered}
$$

And from this we have the proof.

We continue with the case ii) of the general problem: $A(x ; y)=a x^{2}+b y+c$. In this case, we need condition: $a, b, c \geq 0$. If $b=0$, the problem have been considered, For the case $a=0$, it is easy to show that Proposition 2.8 For $b, c \geq 0$ we have

$$
\sqrt{b \sum_{i=1}^{n} x_{i}+c} \leq \sum_{i=1}^{n} \sqrt{b x_{i}+c} \leq \sqrt{n b \sum_{i=1}^{n} x_{i}+n^{2} c} .
$$

Thus we need only considering the case $a, b>0$ :

Theorem 2.9 i) $F_{2}(x ; y) \geq \sqrt{a(x+y)^{2}+2 b(x+y)+4 c}$ if $b^{2}-4 a c \leq 0$. 
ii) $F_{n} \geq \sqrt{\frac{a}{n}\left(\sum_{i=1}^{n} x_{i}\right)^{2}+b \sum_{i=1}^{n} x_{i}+c}+(n-1) \sqrt{c}$.

Proof. By changing variables again, we can assume that $a=b=1$. Then

i) By squaring

$$
\begin{gathered}
\left(\sqrt{x^{2}+y+c}+\sqrt{y^{2}+x+c}\right)^{2}-\left((x+y)^{2}+2(x+y)+4 c\right) \\
=2 \sqrt{y^{2}+c+x} \sqrt{x^{2}+c+y}-x-y-2 c-2 x y
\end{gathered}
$$

and

$$
\begin{gathered}
\left(2 \sqrt{y^{2}+c+x} \sqrt{x^{2}+c+y}\right)^{2}-(x+y+2 c+2 x y)^{2} \\
=(x-y)^{2}(4 c+4 x+4 y-1) \geq 0 .
\end{gathered}
$$

we obtain the result.

ii) We will show that $\sqrt{a}+\sqrt{b} \geq \sqrt{c}+\sqrt{a+b-c}$ for any non-negative number $a, b, c$ with $c=\min (a, b, c)$. Indeed, twice squaring reduces the inequality to $(a-c)(b-c) \geq 0$. From this inequality we have

$$
\begin{aligned}
F \quad & \sum_{i=1}^{n} \sqrt{x_{i}^{2}+x_{i+1}+c} \\
& \geq \sqrt{c}+\sqrt{x_{1}^{2}+x_{2}^{2}+x_{2}+x_{3}+c}+\sum_{i=3}^{n} \sqrt{x_{i}^{2}+x_{i+1}+c} \\
& \geq \quad \ldots \\
& \geq(n-1) \sqrt{c}+\sqrt{\sum_{i=1}^{n} x_{i}^{2}+\sum_{i=1}^{n} x_{i}+c} \\
& \geq \quad(n-1) \sqrt{c}+\sqrt{\frac{1}{n}\left(\sum_{i=1}^{n} x_{i}\right)^{2}+\sum_{i=1}^{n} x_{i}+c .}
\end{aligned}
$$

We have the proof.

\section{Cyclic inequality with power $\frac{1}{3}$}

Let us consider the expression $f(x, y)=a x^{3}+b x^{2} y+c x y^{2}+d y^{3}$. In case of $a+b+c+d=0$, we know that there exists $\alpha, \beta, \gamma$ such that $a x^{3}+b x^{2} y+c x y^{2}+d y^{3}=(x-y)\left(a x^{2}+(a+b) x y-d y^{2}\right)$. However, if $a+b+c+d \neq 0$ we have

Lemma 3.1 For $a, b, c, d, x, y$ are real numbers such that $a+b+c+d \neq 0$, there exist unique $\alpha, \beta, \gamma, \delta$ such that the equality 


$$
a x^{3}+b x^{2} y+c x y^{2}+d y^{3}=(\alpha x+\beta y)^{3}+(\gamma x+\delta y)(x-y)^{2}
$$

holds.

Proof. The right hand side of (3.1) is equal to

$$
(\alpha x+\beta y)^{3}+\left(\delta x^{3}+(\delta-2 \gamma) x^{2} y+(\gamma-2 \delta) x y^{2}+\delta y^{3}\right) .
$$

Hence, by comparing the coefficients of both sides the equality of (3.1) we need to show that the following system has a unique solution

$$
\left\{\begin{array}{c}
\alpha^{3}+\gamma=a \\
3 \alpha^{2} \beta+\delta-2 \gamma=b \\
3 \alpha \beta^{2}+\gamma-2 \delta=c \\
\beta^{3}+\delta=d
\end{array}\right.
$$

Indeed, by eliminating $\gamma, \delta$ from the equations in (3.2) we easily get

$$
\left\{\begin{array}{c}
\alpha+\beta=\sqrt[3]{a+b+c+d} \\
(2 \alpha-\beta)(\alpha+\beta)^{2}=b+2 a-d \\
(2 \beta-\alpha)(\alpha+\beta)^{2}=c+2 d-a .
\end{array}\right.
$$

So we have

$$
\left\{\begin{array}{l}
\alpha=\frac{1}{3}\left(D+\frac{b+2 a-d}{D^{2}}\right)=\frac{3 a+2 b+c}{3 D^{2}} \\
\beta=\frac{1}{3}\left(D+\frac{c+2 d-a}{D^{2}}\right)=\frac{b+2 c+3 d}{3 D^{2}}
\end{array}\right.
$$

where, for simplicity, we put $D=\sqrt[3]{a+b+c+d}$. From the firts and fourth equations of (3.2) we have

$$
\left\{\begin{array}{l}
\gamma=a-\left(\frac{3 a+2 b+c}{3 D^{2}}\right)^{3} \\
\delta=d-\left(\frac{b+2 c+3 d}{3 D^{2}}\right)^{3}
\end{array}\right.
$$

The lemma is proved.

We now want to establish a cyclic inequality between the function

$$
G=\sum_{k=1}^{n} \sqrt[3]{a x_{k}^{3}+b x_{k}^{2} x_{k+1}+c x_{k} x_{k+1}^{2}+d x_{k+1}^{3}},
$$

and the sum $\sum_{i=1}^{n} x_{i}$ for non-negative numbers $x_{1}, x_{2}, \ldots, x_{n}$ with $x_{n+1}=x_{1}$.

Theorem 3.2 Suppose for $a, b, c, d$ be real numbers whose sum is different from 0 , and $27 a(a+b+c+d)^{2} \geq(3 a+2 b+c)^{3}, 27 d(a+b+c+d)^{2} \geq(b+2 c+3 d)^{3}$ then 


$$
G \geq \sqrt[3]{a+b+c+d} \sum_{k=1}^{n} x_{k}
$$

for all $x_{1}, x_{2}, \ldots, x_{n} \geq 0$ with $x_{n}=x_{1}$. We have the reverse inequality

$$
G \leq \sqrt[3]{a+b+c+d} \sum_{k=1}^{n} x_{k}
$$

if

$$
27 a(a+b+c+d)^{2} \leq(3 a+2 b+c)^{3}, 27 d(a+b+c+d)^{2} \leq(b+2 c+3 d)^{3} .
$$

Proof. The proof can be implied directly from the Lemma 3 by noting that with these assumptions we have $\gamma, \delta \geq 0$.

\section{Acknowledgements}

This research is funded by the VNU University of Science under project number TN.16.30. The authors are grateful for this support.

\section{References}

[1] V. Cirtoaje, Algebraic Inequalities: Old and New methods, GIL Publishing House (2006)

[2] T. Tanriverdi, Reformulation of Shapiro's inequality, International Mathematical Forum, Vol. 7, 2012, no. 43, $2125-2130$.

[3] Z. Cvetkovski, Inequalities: Theorems, Techniques and Selected Problems, Springer (2012).

[4] G. Hardy, J. Littlewood and G. Polya: Inequalities, Cambridge University Press, Cambridge (1988).

[5] N.V. Luong, P.V. Hung, N.N Thang: Lectures on Cauchy inequalities, Hanoi National University Publisher (2014) (in Vietnamese).

[6] D.S. Mitrinovic, E.S. Barnes, D.C.B. Marsh, J.R.M. Radok: Elementary inequalities, P. Noordhoff LTD Groningen - The Netherlands (1964)

[7] D.S. Mitrinovic, J.E. Pecaric, A.M. Fink: Classical and New Inequalities in Analysis, Kluwer Academic Publishers (1993).

[8] B.G. Pacpatte: Mathematical inequalities, Elsevier (2005). 